\title{
Optoelectronic Recirculating Delay Line Implementation of a High-Q Optoelectronic Oscillator
}

\author{
G. Charalambous, G.K.M. Hasanuzzaman, A. Perentos and S. Iezekiel \\ Department of Electrical \& Computer Engineering, University of Cyprus \\ Nicosia 1678, Cyprus \\ E-mail: s.iezekiel@ieee.org
}

\begin{abstract}
A dual-loop optoelectronic oscillator incorporating an optoelectronic recirculating delay line in order to circumvent the limitations of optical coherence associated with all-optical loops is demonstrated. The optoelectronic oscillator produces a very stable signal at $5.4 \mathrm{GHz}$ (suitable for WLAN 802.11n and 4GWiMAX systems) with a sub-Hz 3-dB bandwidth and a Q-factor in excess of $10^{10}$. A phase noise of $-115 \mathrm{dBc} / \mathrm{Hz}$ is recorded at 10 $\mathrm{kHz}$ offset, owing to the reduction in phase induced intensity noise, whilst side modes are significantly suppressed for offset values in the range $100 \mathrm{kHz}-1 \mathrm{MHz}$.
\end{abstract}

Index Terms-optoelectronic oscillator; optoelectronic recirculating delay line; infinite impulse response filter; microwave photonics; Q-factor.

\section{INTRODUCTION}

$\mathrm{H}$ Igh purity local oscillators are essential components in many microwave systems. Approaches based on conventional low $\mathrm{MHz}$-frequency electronic oscillators followed by frequency multiplication suffer from a phase noise degradation of $6 \mathrm{~dB}$ with each doubling of frequency [1]. In contrast, photonic-based microwave signal generation using optoelectronic oscillators (OEOs) has been shown to provide superior phase noise performance to conventional microwave oscillators [2].

The simplest OEO consists of a single hybrid loop comprising a relatively long single mode fiber (acting as a highQ energy storage element) with the associated electro-optic and optoelectronic conversions. However, this approach suffers from multiple closely-spaced side modes due to the small free spectral range (FSR) resulting from fiber lengths that are typically several $\mathrm{km} \mathrm{[3].} \mathrm{In} \mathrm{order} \mathrm{to} \mathrm{simultaneously} \mathrm{suppress} \mathrm{the}$ side modes and maintain a high Q-factor, alternative OEO topologies such as the dual-loop configuration [4] have been considered, in which the optical part is split into parallel pathsthe longer loop provides a high Q- factor (but small FSR) and the shorter loop offers a large FSR. Injection-locking techniques can also be considered for further enhancement of the Q-factor [5].

Several OEOs have been recently demonstrated in which microwave photonic filters are used instead of narrowband electrical bandpass filters to achieve both high Q-factor and high side-mode suppression [6], [7]. In line with this trend, we have demonstrated an OEO based on the cascade of a recirculating delay line (RDL) with a dual-loop section [8] as shown in Fig.1 (a).

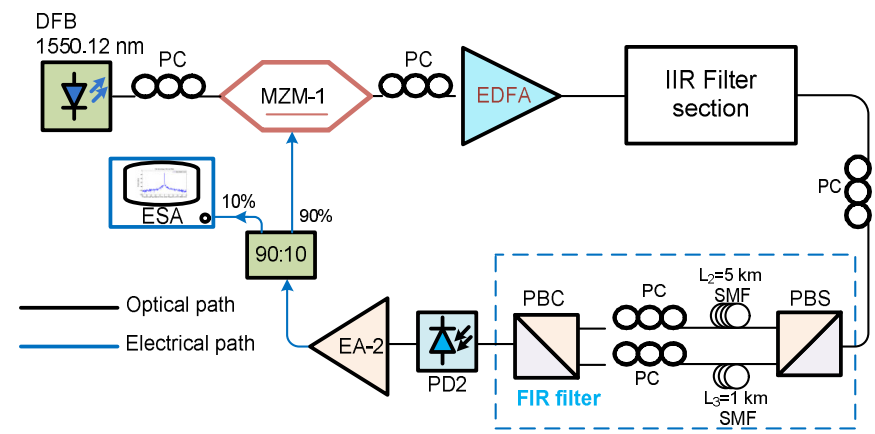

(a)

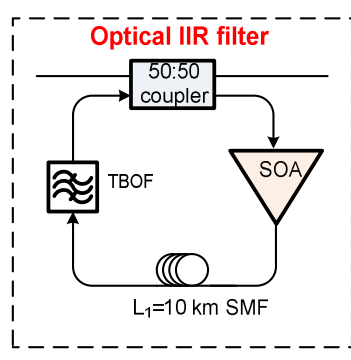

(b)

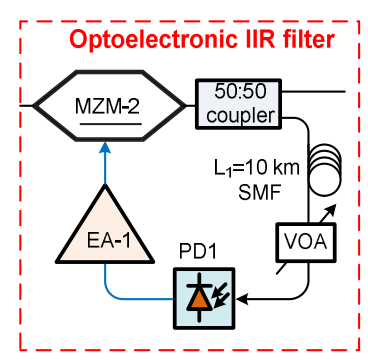

(c)
Fig. 1. (a) Schematic diagram of the experimental OEO. (b) The active optical IIR section of the previous OEO [8]. (c) The optoelectronic IIR section of the OEO implemented in this study. PC: Polarization Controller, SMF: Single Mode Fiber, ESA: Electrical Spectrum Analyzer.

The RDL in [8] was implemented as an active optical loop comprising a fiber delay with an in-line semiconductor optical amplifier (SOA) and tunable bandpass optical filter (TBOF) as indicated in Fig. 1(b). The active RDL acts as an IIR (infinite impulse response) microwave photonic filter and enhances the Q-factor of the dual-loop section due to the production of an 
infinite number of taps, in contrast to previous approaches based on ASE sources which degrade the Q-factor of the OEO [7]. By choosing the RDL delay time to be greater than the coherence time of the laser, incoherent operation can be maintained. Due to wavelength shifting resulting from cross gain modulation (XGM) and to the negative tap generated from the SOA inside the loop, interference phenomena are significantly minimized at the coupler's output. Thus the OEO we reported provides a stable oscillation and a Q-factor of over $10^{10}$. However, the demonstrated phase noise and the SNR (even in the incoherent regime) are not optimal. This is attributed to the excessive amount of phase-induced intensity noise (PIIN) generated due to the multiple delayed optical signals beating at the photodetector, thus limiting the practical application of fiber optic delay line signal processors to OEOs.

The undesirable impact of PIIN can be compensated by using a different wavelength source [9] or using a photodetector [10] for each of the different delayed optical signals. These techniques require numerous optical sources or photodetectors while they alter the conventional fiber optical link configuration in order to combine a high resolution signal processor. A simpler architecture can be found in [11] where a phase modulator of the optical carrier mitigates the phase noise of the information band at higher frequency bands, which can be filtered from the photodetector bandwidth without altering the signal processing link's topology.

Incoherent fiber optic delay signal processors generate PIIN, which can be expressed in terms of two independent contributions [12], [13]. The first depends on the optical source characteristics while the other depends on the delay line signal processor configuration. Therefore, altering the delay line signal processor topology, it is possible to circumvent optical coherence and overcome PIIN by implementing the RDL IIR microwave photonic filter section with an optoelectronic feedback loop as reported in [14], an approach which was also used by us in a bilaterally coupled OEO [15]. This obviates the need for an optical amplifier, thus avoiding any further increases to the ASE noise in the system and significantly suppressing the dominant PIIN.

In this paper we demonstrate a dual-loop based OEO cascaded with a RDL which instead of being implemented with an optically amplified IIR section as in [8], is realized with the optoelectronic loop approach [14]. The optoelectronic loop (as shown in Fig. 1 (c)) is essentially a hybrid loop. Since the resulting OEO does not employ optical amplification in the RDL, the phase noise characteristics and signal to noise ratio (SNR) outperform the previously reported ones [8] whilst other characteristics, such as the Q-factor are maintained. By transforming the active RDL to an optoelectronic loop, the coherence problems and hence the PIIN are reduced owing to the electrical signal modulation.

\section{EXPERIMENTAl SetuP \& OPERATING PRINCIPLE}

The proposed OEO was implemented as illustrated in Fig. 1 (a), with the same overall architecture as [8]; however, the IIR section of that OEO, as shown in Fig. 1(b), was replaced with the optoelectronic IIR section as depicted in Fig. 1(c) in order to remove the requirement for an optical amplifier and to further suppress the PIIN, allowing the use of a high resolution delay line signal processor with improved SNR.

With reference to Fig. 1(a), a quadrature biased MachZehnder modulator (MZM-1) with $V_{\pi}=5.1$ modulates the output of a distributed feedback laser (DFB) emitting at a wavelength of $\lambda_{1}=1550.12 \mathrm{~nm}$ with a $2 \mathrm{MHz}$ linewidth, -140 $\mathrm{dB} / \mathrm{Hz}$ relative intensity noise (RIN) and $11 \mathrm{dBm}$ power which is subsequently amplified via an EDFA. The amplified lightwave then enters the IIR section as detailed in Fig. 1(c), which is implemented with an optoelectronic recirculating delay line (RDL) loop comprising a Mach-Zehnder modulator (MZM-2), a 50:50 coupler, a single mode fiber (SMF), a variable optical attenuator (VOA), a photodetector (PD1) and an electrical amplifier (EA-1). Within the IIR loop, MZM-2 receives the amplified signal from the EDFA and re-modulates it as follows. The output of MZM-2 is split in two paths using a 50:50 coupler. One path of the optical 50:50 coupler is used as an electrical feedback signal to MZM-2 after being delayed, converted to the electrical domain and amplified via a SMF of $10 \mathrm{~km}$ length $\left(L_{1}\right)$, a photodetector (PD1) and an electrical amplifier (EA-1) respectively. Since the feedback signal of the filter section is in the electrical domain, the potential summation of optically coherent signals no longer exists in the IIR section, thereby eliminating interference effects. A VOA is also placed inside the IIR loop to adjust the gain of the filter to be almost equal to $0 \mathrm{~dB}$ in order to operate in a stable regime but with relatively high finesse, as discussed in [14].

The IIR section of the OEO implemented here does not employ any optical amplifiers (SOA or EDFA) and is therefore free from ASE noise. It does however still require in-loop amplification, which is provided via the microwave amplifier EA-1, to overcome the insertion loss of the optical fiber length $L_{1}$ and any losses due to MZM-2. On the other hand, by increasing the optical power level at the input to MZM-2 using the EDFA that precedes the IIR section, it is possible to mitigate loop losses since the electro-optic conversion of the quadraturebiased Mach-Zehnder modulator scales with the input optical power. This EDFA is also used to overcome any additional optical insertion loss due to MZM-2. Even with these considerations taken into account, we have found experimentally that this configuration leads to significantly lower phase noise compared to the OEO implemented with a SOA-based RDL IIR section [8].

The other output of the 50:50 coupler is fed to the dual-loop Finite Impulse Response (FIR) section where it is split into two different delay paths via a polarization beam splitter (PBS). Two SMF delay lines of lengths $L_{2}=5 \mathrm{~km}$ and $L_{3}=1 \mathrm{~km}$ are connected at the output ports of the PBS. Consequently the two signals, which are recombined with a polarization beam combiner $(\mathrm{PBC})$, are free from any interference from each 
other. The combined signals are then converted to the electrical domain by a high speed photodiode (PD2) and the generated RF output signal is then passed through a low noise microwave amplifier before being fed to the RF port of MZM-1, thus closing the complete OEO loop. The low noise amplifier is realized by cascading two broadband amplifiers (of noise figure $8 \mathrm{~dB}$ and $7 \mathrm{~dB}$ respectively). Polarization controllers (PC) are used at various points in the system: before MZM-1, EDFA, MZM-2, PBS and PBC, the last two to tune the power distribution and correct the polarization deviations caused by the SMF.

\section{RESULTS \& DISCUSSION}

The measured electrical spectrum of the proposed dual-loop optoelectronic recirculating delay line based OEO is shown in Figs 2(a)-2(d) at four different spans. The center frequency of the oscillating signal is $5.4 \mathrm{GHz}$; this specific value is determined by the fiber delay line lengths $\left(L_{1}, L_{2}\right.$ and $\left.L_{3}\right)$ from the IIR and FIR filter sections as analyzed in [8].

The quality factor of the oscillation frequency, as shown in Fig. 2(d), was measured with an electrical spectrum analyzer (ESA) with a span of $500 \mathrm{~Hz}$ and a resolution bandwidth (RBW) of $3 \mathrm{~Hz}$. The system is not sensitive to vibrations or polarization variations due to the incoherent operation of the signal processors. The oscillation frequency drift is limited up to $5 \mathrm{~Hz} / \mathrm{s}$ (outside of frequency hopping, which is due to bias drift of the MZMs). The signal track function of the ESA keeps the signal centered on the display while the signal drifts. The 3$\mathrm{dB}$ bandwidth $\Delta f_{3 \mathrm{~dB}}$ was found to be less than $1 \mathrm{~Hz}$ yielding a Q-factor greater than $10^{10}\left(Q=f_{o s} / \Delta f_{3 \mathrm{~dB}}\right)$. The side mode suppression ratio was measured at $60 \mathrm{~dB}$.
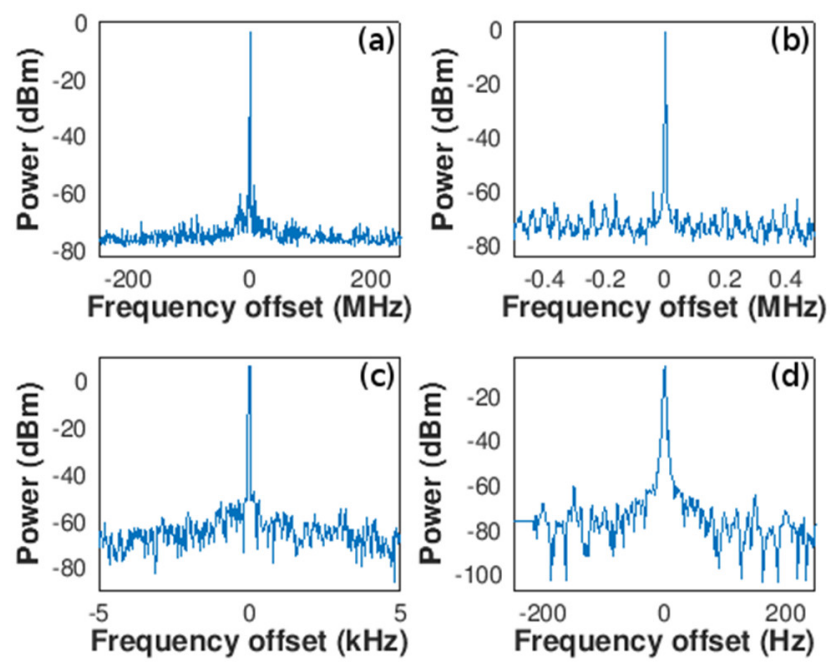

Fig. 2. Electrical spectra of the generated $5.4 \mathrm{GHz}$ oscillation at (a) RBW: $10 \mathrm{kHz}$, SPAN: $500 \mathrm{MHz}$ (b) RBW: $1 \mathrm{kHz}$, SPAN: 1 MHz (c) RBW: $30 \mathrm{~Hz}$, SPAN: $10 \mathrm{kHz}$ (d) RBW: $3 \mathrm{~Hz}$, SPAN: $500 \mathrm{~Hz}$.
The performance of this OEO was then compared with that from [8]; Fig.3 shows the spectra of the two generated oscillations using an active optical RDL and an optoelectronic loop. The noise floor of the oscillation signal from the OEO employing the optoelectronic loop IIR filter (red line) has been reduced by $10 \mathrm{~dB}$ compared to that of the OEO used in [8]. For this comparison, all OEO parameters except for the IIR section were kept constant. The active RDL implementation of the IIR section that was used is identical to that described in [8].

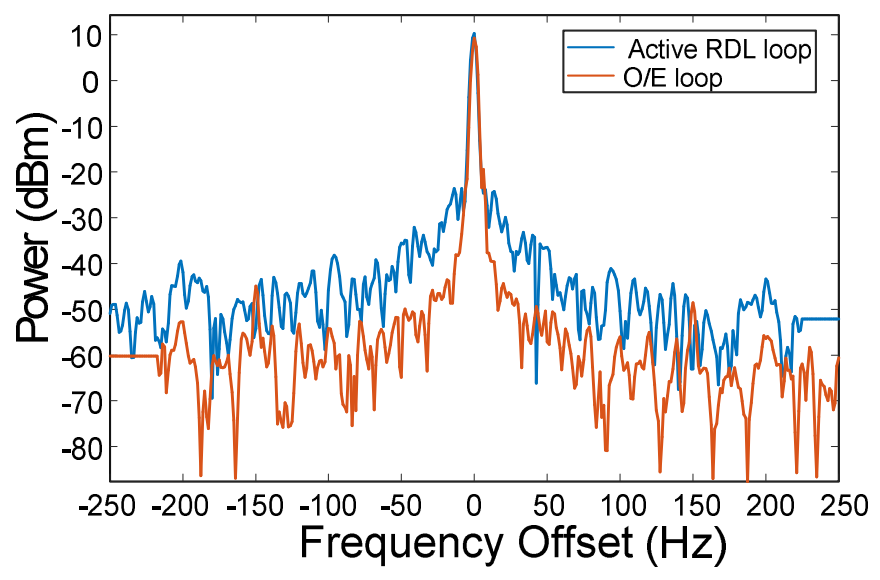

Fig. 3. Electrical spectra of the generated $5.4 \mathrm{GHz}$ oscillation for the active optical RDL loop as implemented in [8] (blue line) and the O/E loop implemented here (red line). RBW: $3 \mathrm{~Hz}$, SPAN: 500 $\mathrm{Hz}$.

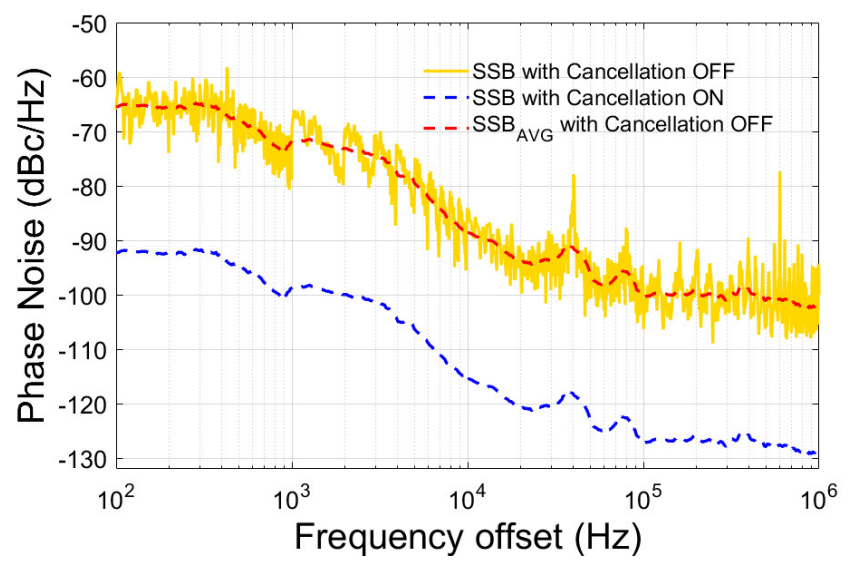

Fig. 4. Single side-band (SSB) phase noise measurement of the generated microwave signal at $5.4 \mathrm{GHz}$.

The single side-band phase noise measurement of the proposed OEO is shown in Fig.4; the phase noise was initially measured as being $-88 \mathrm{dBc} / \mathrm{Hz}$ and $-100 \mathrm{dBc} / \mathrm{Hz}$ at $10 \mathrm{kHz}$ and $1 \mathrm{MHz}$ offset from the carrier respectively, using direct measurement from the spectrum analyzer. However, the direct spectrum method cannot accurately measure phase noise levels below $-101 \mathrm{dBc} / \mathrm{Hz}$ at low frequency offsets $(<10 \mathrm{kHz})$. The displayed result is the combination of the noise of the input signal, the internal noise of the analyzer (triggered by the input 
signal) and the displayed average noise level of the analyzer (DANL) which is the amplitude of the analyzer's noise floor over a given frequency range [16]. The DANL is included in the measurement regardless of the signal being present or not and limits the range over which the analyzer can measure phase noise because the DUT phase noise is below the noise floor of the analyzer at large frequency offsets. Therefore, DANL becomes significant at frequency offsets from the carrier of 1 $\mathrm{MHz}$ to $10 \mathrm{MHz}$ [16]. Thus to further improve the measurement of the device under test (DUT), we used the cancellation method of the analyzer's phase noise personality (option 226) which removes the contribution of the analyzer's internal noise [16], [17]. This is done by comparing a stored reference measurement with the measured phase noise of the device under test. After using the cancellation method, the phase noise is measured as $-115 \mathrm{dBc} / \mathrm{Hz}$ at $10 \mathrm{kHz}$ and around $-128 \mathrm{dBc} / \mathrm{Hz}$ at $1 \mathrm{MHz}$ offset. Compared to the optical IIR filter of [8], the optoelectronic IIR filter reduces the phase noise by $14 \mathrm{~dB}$ at an offset of $10 \mathrm{kHz}$ (including the internal analyzer's noise). This reduction of phase noise is due to the significant suppression of the PIIN, elimination of the SOA and the associated ASE in the IIR section thereby indicating the potential of an optoelectronic recirculating delay line to suppress noise.

\section{CONCLUSION}

We have demonstrated a high-Q dual-loop OEO using an optoelectronic RDL for the IIR filter section. Although it contains more active components than [8], the demonstrated OEO achieved an enhanced phase noise performance compared to our previously reported OEO where the IIR filter section was implemented with an active all-optical loop. In particular, the PIIN and phase noise have been reduced by using electrical feedback in the loop as a means to avoid optical coherence (and hence interference) problems. A Q-factor of over $10^{10}$ has been maintained with phase noise improvement of $41 \mathrm{dBc}$ at $10 \mathrm{kHz}$ (cancelling the contribution of the internal analyzer's noise) and a noise floor improvement of $10 \mathrm{~dB}$. It is expected that the phase noise performance could be further improved through the use of zero dispersion fiber, for example [18].

\section{ACKNOWLEDGMENT}

This project has received funding from the European Union's Horizon 2020 research and innovation programme under the Marie Skłodowska-Curie grant agreement No 642355. This work also falls under the Cyprus Research Promotion Foundation's Framework Program for Research, Technological Development and Innovation, co-funded by the Republic of
Cyprus and the European Regional Development Fund, and specifically under Grant $\triangle \mathrm{IAKPATIKE} \Sigma / \mathrm{KY}-\mathrm{I} \Sigma \mathrm{P} / 0114$.

\section{REFERENCES}

[1] J. A. Nanzer et al., "Photonic Signal Generation for Millimeter Wave Communications", Johns Hopkins APL Technical Digest, vol. 30, no. 4, 2012

[2] X. Yao and L. Maleki, "Optoelectronic microwave oscillator," J. Opt. Soc. Amer. B, vol. 13, no. 8, pp. 1725-1735, Aug. 1996.

[3] L. Maleki, "The optoelectronic oscillator," Nat. Photonics, vol. 5, no. 12, pp. 728-730, Dec. 2011.

[4] X.S. Yao and L. Maleki, "Multiloop optoelectronic oscillator," IEEE J. Quantum Electron., vol. 36, no. 1, pp. 79-84, Jan. 2000.

[5] W. Zhouand and G. Blasche, "Injection-locked dual opto-electronic oscillator with ultra-low phase noise and ultra-low spurious level," IEEE Trans. Microw. Theory Tech., vol. 53, no. 3, pp. 929-933, Mar. 2005.

[6] F. Jiang et al., "An optically tunable wideband optoelectronic oscillator based on a bandpass microwave photonic filter,'Opt. Express, vol. 21,no. 14, pp. 16381, July 2013.

[7] X. Liu et al., "A reconfigurable optoelectronic oscillator based on cascaded coherence-controllable recirculating delay lines," Opt. Express, vol. 20, no. 12, pp. 13296, May 2012.

[8] G. Charalambous, A. Perentos and S. Iezekiel, "High-Q Optoelectronic Oscillator based on Active Recirculating Delay Line and Dual-loop Topology," IEEE Photonics Technology Letters, vol. 28, no. 24, pp. 2850-2853, Dec. 2016.

[9] C. Pulikkaseril, E. H. W. Chan and R. A. Minasian, "Coherence-Free Microwave Photonic Bandpass Filter Using a Frequency-Shifting Recirculating Delay Line," Journal of Lightwave Technology, vol. 28, no. 3, pp. 262-269, Feb. 2010.

[10] M. Frankel and R. Esman, "Fiber-optic tunable microwave transversal filter", IEEE Photonics Technology Letters, vol. 7, no. 2, pp. 191-193, 1995.

[11] E. Chan, "Suppression of phase-induced intensity noise in fibre optic delay line signal processors using an optical phase modulation technique", Optics Express, vol. 18, no. 21, p. 21573, 2010.

[12] B. Moslehi, "Analysis of optical phase noise in fiber-optic systems employing a laser source with arbitrary coherence time", Journal of Lightwave Technology, vol. 4, no. 9, pp. 1334-1351, 1986.

[13] M. Tur and A. Arie, "Phase induced intensity noise in concatenated fiber-optic delay lines", Journal of Lightwave Technology, vol. 6, no. 1, pp. 120-130, 1988.

[14] L. Cheng and S. Aditya, "A Novel Photonic Microwave Filter With Infinite Impulse Response", IEEE Photonics Technology Letters, vol. 19, no. 19, pp. 1439-1441, Oct. 2007.

[15] G. Charalambous, G.K.M. Hasanuzzaman, A. Perentos and S. Iezekiel, Ultra-high-Q Optoelectronic Oscillator based on Bilaterally Coupled Loops, in Proc. IEEE MWP2016, International Topical Meeting on Microwave Photonics, Long Beach, USA, Nov. 2016.

[16] Literature.Cdn.Keysight.Com. (2016). http://literature.cdn.keysight.com/litweb/pdf/E444090233.pdf?id=129 4529 (accessed 13 December 2016).

[17] http://literature.cdn.keysight.com/litweb/pdf/5988-4348EN.pdf (accessed 20 October 2016)

[18] K. Volyanskiy, Y. K. Chembo, L. Larger, and E. Rubiola, "Contribution of Laser Frequency and Power Fluctuations to the Microwave Phase Noise of Optoelectronic Oscillators," J. Lightw. Technol., vol. 28, no. 18, pp. 2730-2735, Sept. 2010. 